\title{
Continuous high power microwave heating at the W7-X stellarator
}

\author{
K.J. Brunner ${ }^{1}$, H. Braune ${ }^{1}$, V. Erckmann ${ }^{1}$, W. Kasparek ${ }^{1}$, H.P. Laqua ${ }^{1}$, S. Marsen ${ }^{1}$, \\ D. Moseev ${ }^{1}$, B. Plaum ${ }^{2}$, T. Stange ${ }^{1}$, M. Weißgerber ${ }^{1}$ and the entire W7-X team \\ ${ }^{1}$ Max-Planck-Institute for Plasma Physics, Greifswald/Garching, Germany, k.j.brunner@ipp.mpg.de \\ ${ }^{2}$ IGVP Stuttgart University, Stuttgart, Germany
}

\section{Introduction}

The W7-X advanced stellarator is a fusion experiment relying heavily on electron cyclotron resonance heating (ECRH). It uses a quasi-optical transmission line able to transmit up to 12 gyrotrons from a remote gyrotron assembly to the plasma vessel [1]. This method of transmission delivers high power densities and low losses at a moderate price tag. The microwave power is eventually coupled into the plasma using movable front steering mirrors, therefore providing a semi-arbitrary choice for the angle of incidence. In addition the incident polarization and ellipticity can be freely set via steerable polarizers in the transmission line. In addition to the frontsteering mirrors the system can divert power to a remote steering launcher via a movable mirror [3].

The first operation phase (OP1.1) was conducted with 6 gyrotrons operating at $140 \mathrm{GHz}$ and supplying a total of $4.3 \mathrm{MW}$ of continuous heating power to the plasma. This setup already proved the capabilities of the system during the first operation phase, yielding outstanding plasma parameters, which will be briefly presented in the first section.

For the second operation phase (OP1.2) envisaged to start in September 2017, four additional gyrotrons have been installed, which will give access to up to $8.5 \mathrm{MW}$ of heating power. This opens up new operation regimes, which also come with new challenges regarding heating efficiency and machine safety. This paper focuses on the developments to the heating system from a plasma physics perspective and will only briefly summarize the hardware developments in section 3. For a more thorough description of the W7-X ECRH hardware developments the reader is kindly referred to the corresponding publications also published as part of these proceedings [2, 3].

\section{Main findings of OP1.1}

The application of ECRH in fusion plasmas extends beyond plain bulk plasma heating, even though this is its main purpose. Examples for other applications wall conditioning, plasma profile shaping, instability control and electron cyclotron current drive (ECCD). During OP1.1 many of these aspects were already tested.

One of the outstanding results of this operation phase was the very high central electron temperature approaching $10 \mathrm{keV}$. These temperatures are achieved using $2^{\text {nd }}$ harmonic X-mode heating (X2), which has a very narrow power deposition profile in the plasma core. The density in these discharges remained at a few $10^{19} \mathrm{~m}^{-3}$, which also resulted in moderate ion temperatures at roughly $2 \mathrm{keV}$ due to the low collisional coupling between the electrons and ions. An example of such a discharge is seen in figure 1, which shows shot number 20160301.014.
The core electron cyclotron emission (ECE) temperature can be seen in the middle plot.

A second prominent result from OP1.1 are the ECCD-induced core temperature crashes observed. The shot shown in figure 1 depicts a shot exhibiting this phenomenon as well. The top graph shows injected ECRH power per gyrotron totalling about $2 \mathrm{MW}$. The integrated toroidal plasma current measured by Rogowski coils is plotted at the bottom. The current drive was chosen to increase the rotational transform (Co-ECCD). Total X2 absorption is reached within a few tens of milliseconds of this shot and stays this way throughout. After $150 \mathrm{~ms}$ the then quasi-steady core electron temperature $T_{e}$ is suddenly dropping sharply and recovers. The onset of this phenomenon coincides with the rise in measured $\mathrm{I}_{\text {tor }}$. These sawtooth-instability-like crashes are likely the result of the iota profile changing, thus resulting in the emerging of instabilities at rational flux surfaces [4].

\section{Developments for OP1.2}

As has been mentioned before this paper will not elaborate on the ECRH hardware developments. In summary there will be a significant increase in available heating power up to $8.5 \mathrm{MW}$, which is supplied by 10 gyrotrons for up to $30 \mathrm{~min}$. In addition the fueling capabilities of W7-X have been extended by a pellet injector for "deep fueling". The combination of higher fueling and increased heating power is expected to give access to plasma densities in the $10^{20} \mathrm{~m}^{-3}$ range. This however poses the problem that the $\mathrm{X} 2$ heating scheme, which has been predominantly employed during OP1.1, will suffer from significant beam refraction effects as the $\mathrm{X} 2$ cut-off density of $1.2 \cdot 10^{20} \mathrm{~m}^{-3}$ is approached. For this reason the OP1.2 phase of the $\mathrm{W} 7-\mathrm{X}$ experiment will focus on optimizing the $2^{\text {nd }}$ harmonic ordinary mode $(\mathrm{O} 2)$ heating scheme, the cut-off density of which is twice that of the X2 scheme $\left(2.4 \cdot 10^{20} \mathrm{~m}^{-3}\right)$.

This heating scheme however has a lower single pass absorption efficiency. Where X2 heating easily reaches $100 \%$ even at low temperatures, the $\mathrm{O} 2$ absorption efficiency, which scales with $\mathrm{T}_{\mathrm{e}}^{2}$, will barely exceed $60 \%$ single-pass absorption below a core electron temperature of $2 \mathrm{keV}$. For this reason a set of Tungsten-coated (TZM) holographic tiles have been installed on the wall opposite to the ECRH launchers. These have been designed to refract about $90 \%$ of the incident ECRH power back through the magnetic axis. This setup is complemented by a non-holographic polished steel liner on the outboard side, which serves the same purpose. The plasma center exhibits the highest electron temperature and therefore the total absorbed power fraction is expected to increase from $60 \%$ to well above $80 \%$ as indicated by figure 2 . This will be verified in OP 1.2 . 
The TZM tiles are an integral part of the efficient multi-pass $\mathrm{O} 2$ heating scheme and as such it is crucial, that the ECRH heating beam is well aligned on the tile's refraction surface.

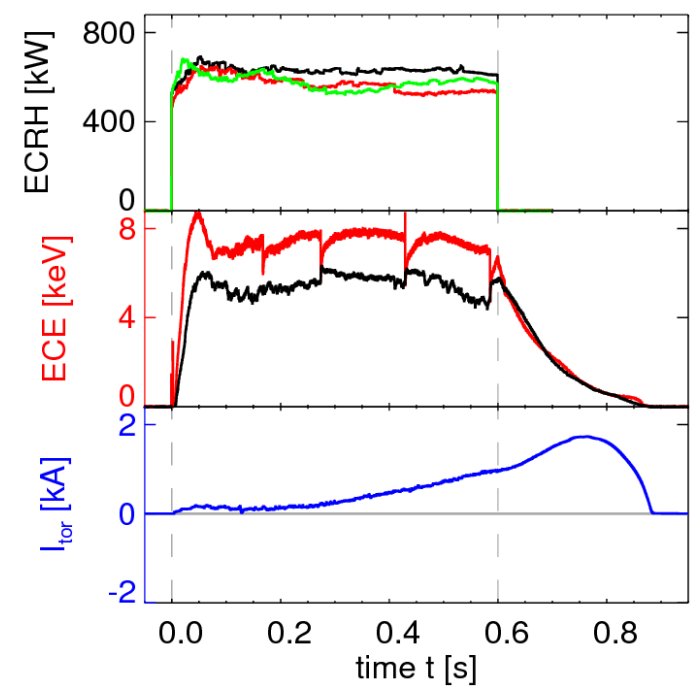

Fig. 1. W7-X shot number 20160301.014. The top shows the inserted ECRH power for each gyrotron totalling roughly 2 MW. Below are the radiative temperature measured by ECE for the central channel (red) and the channel totalling $\mathrm{r} / \mathrm{a}=0.4$. The measured toroidal plasma current is at the bottom. The crashes of the central electron temperature as a result of CoECCD indicates the presence of current driven plasma instabilities (adapted from [5])

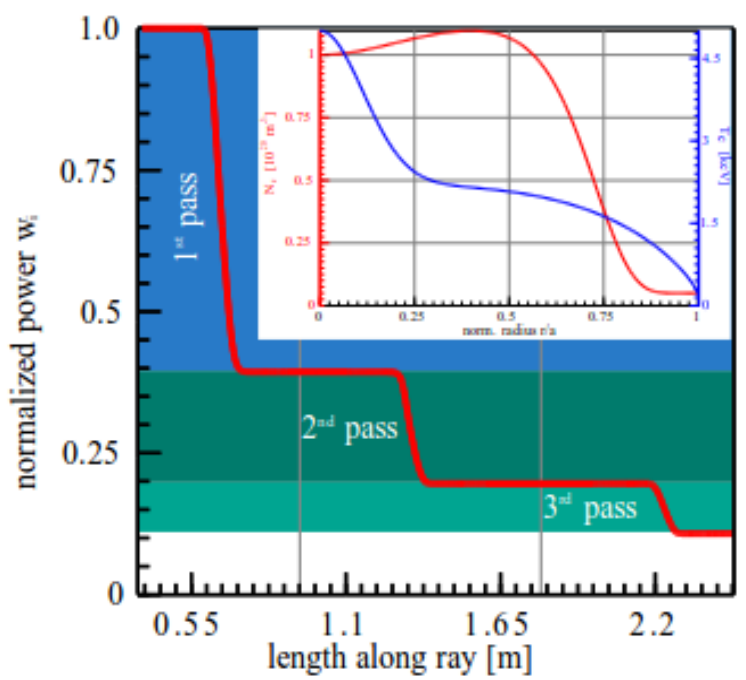

Fig. 2.TRAVIS simulation of ECRH absorption efficiency for a triple-pass through the W7-X plasma under optimum conditions. In the first pass only $60 \%$ of the ECRH power are absorbed, which can be increased significantly by adding the second and third pass. The assumed plasma parameter profiles can be seen in the inlay
However, even though the $\mathrm{O} 2$ beam is not expected refract a lot at the medium plasma densities expected during OP1.2, the amount of refraction suffered is still sufficient to lose about $50 \%$ of the beam at the tile. For this reason it is necessary to implement a feedback targeting system for the launcher mirrors. This will initially be implemented in a feed-forward scheme using the TRAVIS code [6]. In addition a set RF probing holes have been added to the TZM tiles, which will be used to measure the shine-through power of the ECRH beam incident on the tile. Since there is generally more than 1 probing hole, one can use this diagnostic as a feedback system controlling the beam position on the tile. This will be tested during OP1.2. To complement this diagnostic the infrared (IR) camera system observing the inboard plasma wall from the ECRH launchers has been improved to be able to detect up to $15 \mu \mathrm{m}$ IR radiation, making the ECRH beam visible on the bare reflector tiles.

\section{Summary}

The ECRH system on W7-X delivered outstanding plasma performance during the initial experimental campaign OP1.1. For the next operation phase the system has been further expanded, giving access to even higher performance plasma regimes, which at the same time pose significant challenges for the operation of the heating system in particular the development of a safe high power $\mathrm{O} 2$ heating scheme at densities well above the $\mathrm{X} 2$ cut-off.

\section{Acknowledgements}

This work has been carried out within the framework of the EUROfusion Consortium and has received funding from the Euratom research and training programme 2014-2018 under grant agreement No 633053. The views and opinions expressed herein do not necessarily reflect those of the European Commission.

\section{References}

1. Erckmann, V. et al. Electron Cyclotron Heating for W7X: Physics and Technology // Fusion Sci. Technol. 2007. V. 52 No. 2 P. 291-312.

2. Braune, $H$. Title to be added // Proc. of the SMP. 2017, P. t.b.d.

3. Kasparek, $W$. Continuous High Power Microwave Heating At The W7-X Stellarator // Proc. of the SMP 2017, P. t.b.d.

4. Estrada, T. et al. Transient behaviour in the plasma core of TJ-II stellarator and its relation with rational surfaces // Pl. Phys. \& Contr. Fus., 2002, V. 44, P. 1615-1624.

5.Stange, T. et al. Advanced electron cyclotron heating and current drive experiments on the stellarator Wendelstein 7-X // Proc. of the RFFPC, 2017, Inv-04.

6. Marushchenko, N.; Turkin, Y. \& Maassberg, H. Raytracing code TRAVIS for ECR heating, EC current drive and ECE diagnostic // Comp. Ph. Comm., 2014, V. 185, P. 165-176. 